\title{
The quark-gluon medium (micro- and macro-QCD)
}

\author{
I.M. Dremin \\ Lebedev Physical Institute, Moscow 119991, Russia
}

\begin{abstract}
The properties of the quark-gluon medium observed in high energy nucleus-nucleus collisions are discussed. The main experimental facts about these collisions are briefly described and compared with data about proton-proton collisions. Both microscopic and macroscopic approaches to their description are reviewed. The chromodynamics of the quark-gluon medium at high energies is mainly considered. The energy loss of partons moving in this medium is treated. The principal conclusion is that the medium possesses some collective properties which are crucial for understanding the experimental observations.
\end{abstract}

PACS numbers: 24.85.+p - 12.38.Mh

\section{Introduction}

The topic of the quark-gluon medium is so widely spread nowadays that it can not be squeezed into a single lecture. That is why I mostly concentrate on general ideas about the evolution of the quark-gluon medium in high energy heavy ion collisions and on those properties of the medium which are revealed by the energy losses of partons moving in it. They are described by the chromodynamics of the quark-gluon medium which is of the main concern in this lecture. It ascribes quarks and gluons as elementary objects (partons) responsible for the interaction. The goal is to confront experimental data and theoretical ideas about the properties of the quark-gluon medium formed in high energy collisions of hadrons and nuclei (for recent review see [1]).

General "consensus" is achieved concerning the types of processes observed in pp-collisions and their description. They are split into the two groups: SOFT (low $p_{T}$ ) processes, where one uses phenomenology, notion of clusters, multiperipheral models, BFKL equations; and HARD (large $p_{T}$ ) processes, where one considers perturbative QCD, jets production and their evolution, DGLAP equations.

\footnotetext{
${ }^{1}$ Email: dremin@lpi.ru
} 
Highly coherent parton configurations and strong internal fields become especially important for the matter in collision. By colliding two heavy nuclei at ultrarelativistic energies one expects to get a hot and dense internally colored medium. It should exhibit some collective properties not seen at static conditions.

\section{The main experimental findings}

Nowadays, high energy experimental data are obtained at the following accelerators: Tevatron $(p \bar{p}, \sqrt{s} \leq 1.96 \mathrm{TeV})$; RHIC (pp and ions (up to $\mathrm{Au}+\mathrm{Au}$ ), $\sqrt{s} \leq 200 \mathrm{~A} \mathrm{GeV}$ ); LHC (pp, $\sqrt{s}=0.9 ; 2.36$; 7 ; planned $14 \mathrm{TeV}$; and $\mathrm{Pb}+\mathrm{Pb}$, $\sqrt{s}=2.76 \mathrm{~A} \mathrm{TeV}$; planned $5.5 \mathrm{~A} \mathrm{TeV})$.

The very first question addressed is the difference in AA and pp processes at high energies. The following characteristics were studied:

1.Ratio of particle spectra in AA and pp

$$
R_{A A}\left(y, p_{T} ; b\right)=\frac{d^{2} N_{A A} / d y d p_{T}}{N_{p a r t} d^{2} N_{p p} / d y d p_{T}}
$$

shows that $R_{A A} \approx 0.2$ at high $p_{T}>4 \mathrm{GeV}$ which indicates jet quenching (both at RHIC and LHC) and leads to conclusion that AA are not reducible to pp.

2. Correlations: two-, three-particle; jets; BE-HBT; ridge; double-humped distributions... They reveal, e.g., collective flows: $v_{2}$ - elliptic (azimuthal) flow induced by the liquid pressure

$$
E \frac{d^{3} N}{d^{3} p}=\frac{1}{2 \pi} \frac{d^{2} N}{d y p_{T} d p_{T}}\left(1+\sum_{n=1}^{\infty} 2 v_{n} \cos \left[n\left(\phi-\Psi_{r}\right)\right]\right)
$$

3. Enhancement: hadrocomposition changes (e.g., strangeness, $\mathrm{p} / \pi$, quarkonia ...)

These facts lead to CONCLUSION: The quark-gluon medium is formed (plasma, liquid).

Many important features were observed in $7 \mathrm{TeV}$ pp-collisions at LHC. For example, particle density in pp (already at $2.36 \mathrm{TeV}$ ) becomes comparable to that of AA at RHIC; the size of the interaction region at high multiplicities becomes comparable to that of AA at RHIC (as found from Bose-Einstein correlations), and, most spectacular, ridge was observed in 
high multiplicity pp at $7 \mathrm{TeV}$. It raises the question whether this is the signature of the quark-gluon medium formed even in pp. No definite answer was found yet.

\section{From partons and fields to the quark-gluon medium}

The evolution of quark-gluon fields at the initial stages of collisions is described by microscopic QCD. Later, due to some instabilities the collective properties of this system develop and a quark-gluon matter is formed. At this stage its properties (in particular, propagation of partons through it) should be describable both by micro- and macro-QCD.

At the very beginning, the two Lorentz-contracted sheets with transverse partonic fields (called the Color Glass Condensate - CGC) collide. Soon afterwards they transform into a system of longitudinal fields in color flux tubes localized in the transverse plane and stretching between the valence color degrees of freedom. It is called Glasma. This evolution is described by the JIMWLK-equation [2] obtained from micro-QCD. It preceeds the next stage where the matter with collective properties is formed (possibly, after some instabilities). It is named the Quark-Gluon Plasma (QGP). At the final stage it hadronizes producing final particles.

The mechanical and thermodynamical properties of QGP are studied by comparison of experimental data with theoretical results obtained in the framework of the QCD-inspired hydrodynamics briefly discussed in part 4 of the lecture.

The energy loss of partons in the quark-gluon medium is the main source of experimental information about its properties during the QGP stage. As in electrodynamics, it may be separated in the two categories.

The loss due to the change of the velocity vector of a parton such as elastic scattering, bremsstrahlung and synchrotron radiation is usually treated microscopically. All these phenomena result from the short-range response of the parton to the impact of the matter fields. Elastic scattering does change the parton energy due to the recoil effect and deflects it and thus changes the energy flow in the initial direction. At high energies it is less probable than the emission of gluons (bremsstrahlung) during the nearby collisions with the matter constituents. The medium structure imposes some conditions on the 
coherence properties of the radiation process and on the effective radiation length (in analogy to the Ter-Mikhaelyan and Landau-Pomeranchuk effects in electrodynamics). In general, the intensity of the radiation depends on the relation on the path length of the parton in the medium, its mean free path (defined by the distance between the scattering centers and by cross sections) and the formation length of emitted radiation. The synchrotron radiation of gluons induced by the curvature of parton trajectory in chromomagnetic fields may become important for strong enough fields. Namely these processes are considered in part 5 of the lecture.

Principally different source of energy losses is connected with the medium polarization by the propagating parton. This is the collective response of the medium related to the non-perturbative long-range interconnection of its constituents described by its chromopermittivity. It is treated macroscopically by the in-medium QCD. The corresponding effects are analogous to the Cherenkov radiation, the wake and the transition radiation. In these processes one can, in the first approximation, neglect the change of the velocity vector of a parton. The macroscopical aspect of the problem is often ignored and I will discuss it in more details in the part 6 of the lecture.

The total energy loss is the sum of the both micro- and macro-losses.

\section{Hydrodynamics (thermodynamical and me- chanical properties of QGP)}

The thermodynamical and mechanical characteristics of QGP change during its evolution. Spatial and temporal information is necessary to get them. The lattice calculations and ideas about CGC, Glasma, QGP provide some hints to transition from confined state to deconfined quarks and gluons. The inelastic collisions may lead to thermalization of the medium. Subsequently, it expands and hydrodynamics may be applied to treat this stage of evolution. It is described in many reviews (e.g., see [3, 4]) and I give just a brief survey of it here.

The medium is characterized by 6 independent variables. Those are the energy density $e$, pressure $p$, baryon number $n_{B}$ and 3 components of the

velocity vector $u_{\mu}$. The energy-momentum tensor and baryon number current are

$$
T^{\mu \nu}(x)=(e(x)+p(x)) u^{\mu}(x) u^{\nu}(x)-p(x) g^{\mu \nu}, \quad j_{B}^{\mu}(x)=n_{B}(x) u^{\mu}(x) .
$$


Six hydrodynamical equations of motion govern these variables. Those are five non-linear partial differential equations obtained from local conservation laws for energy, momentum and baryon number

$$
\partial_{\mu} T^{\mu \nu}(x)=0 \quad(\nu=0, \ldots, 3) ; \quad \partial_{\mu} j_{B}^{\mu}(x)=0
$$

with an equation of state relating $p, e$ and $n_{B}$. It is usually chosen to mimic the lattice QCD results by matching the states below and above the critical temperature (i.e., hadrons to a quark-gluon medium). This is rather indefinite element of the whole approach. Besides, the solutions of the nonlinear equations in $(3+1)$-dimensions ask for initial conditions to be defined and may be only obtained numerically with several external parameters. The shape of initial hydro profile is a major source of uncertainty. That is the origin of several conflicting results. The CGC and Glasma approaches provide some guesses to the initial conditions for the evolution of a thermalized QGP. However, the evolution of the Glasma into a thermalized QGP is not yet understood.

At least four parameters are necessary to fix the initial conditions and freeze-out algorithm which determines the transition from the hydrodynamical characteristics to hadronic stage. Those are the initial time, energy (or entropy) density, baryon number and the freeze-out temperature (or decoupling energy density) $\tau_{e q} ; s_{e q} ; n_{B, e q} ; e_{d e c}$. They are fixed by further comparison with experimental data. Schematically, the correspondence of these parameters and experimental characteristics can be represented as $d N / d p_{T}-$ $T-e_{d e c} ; d N / d y-(\tau s)_{e q} ; p / \pi-n_{B, e q} / s_{e q} ;\left(d N / d p_{T}\right)_{p} /\left(d N / d p_{T}\right)_{\pi}-\tau_{e q}$. Moreover, from the overlap geometry of the two colliding nuclei one should define the shape of the initial density profiles as well as assume the profiles of the initial longitudinal and transverse flows and final hadronization prescriptions.

From solutions of the hydrodynamical equations one gets the transverse momentum spectra for various species of particles, the radial and elliptic flows, the shape of the interaction region (as revealed by Bose-Einstein correlations and Hanburry Brown-Twiss interferometry). Their comparison with experimental data allows to determine the main thermodynamical, statistical and mechanical properties of the quark-gluon medium. Symbolically, their values found from $200 \mathrm{GeV}$ data can be grouped as $T_{e q} \approx 360 \mathrm{MeV}$; $T_{c r} \approx 170 \mathrm{MeV} ; T_{\text {dec }} \approx 120 \mathrm{MeV} ; \tau_{\text {therm }}=\tau_{\text {eq }} \approx 0.6<1 \mathrm{fm} ; \tau_{\text {dec }} \approx 7 \mathrm{fm} ;$ $e_{t h} \approx 25 \mathrm{GeV} / \mathrm{fm}^{3} ; e_{c r} \approx 1 \mathrm{GeV} / \mathrm{fm}^{3} ; e_{\text {dec }} \approx 0.075 \mathrm{GeV} / \mathrm{fm}^{3} ; s_{\text {eq }} \approx 110$ $\mathrm{fm}^{-3} ; \eta / s \approx 0.1(1 / 4 \pi$ in $\mathrm{AdS} / \mathrm{CFT}) ; n_{B}<0.5 \mathrm{fm}^{-3}$. They show rapid 
thermalization, high average initial energy density and quite long "lifetime" of the quark-gluon plasma before hadronization at rather low energy density and temperature predicted by lattice QCD. Complete thermalization in the time less than $1 \mathrm{fm}$ is required to obtain the measured value of elliptic flow and its centrality dependence which is very sensitive to any deviation from local thermal equilibrium of low $p_{T}$ particles. Collective excitations, resonances and inelastic collisions keep the system in thermal equilibrium. The good agreement of the data with ideal fluid dynamics points to a very small viscosity of QGP. Other transport coefficients (shear, diffusion, heat conduction) are not important if the microscale defined by rescattering is much less than the macroscale related to medium expansion. Strong nonperturbative interaction should be responsible for its behavior as an ideal liquid. Herefrom comes the name of strong quark-gluon plasma (sQGP). In sQGP, there may exist, e.g., clusters [5] and colored bound states of massive quasiparticles [6] with heavier flavors. Resonance scattering on constituents of the quark-gluon medium can become important [7]. All that would give rise to its collective response with new scales discussed above, high pressure, large chromopermittivity and long-range correlations necessary to explain the enhancement of strange partons production by non-local processes. The long "lifetime" supports approximate description of energy losses within an "infinite" medium.

Hydrodynamics is an actively developing field nowadays. Main characteristics of low $p_{T}$ particles have been fitted in this approach albeit many factors have to be taken into account such as thermodynamics, models of collective flows, hadronization process, resonance decays, chemical composition, geometry of collisions etc. However a fully consistent hydrodynamical description has not yet been found. Some controversial conclusions appear from time to time about, e.g., energy (from SPS to LHC), rapidity and centrality dependence of elliptic flow, its absolute value, the transverse momentum dependence of various radii derived from HBT analysis, the chemical composition of some species (e.g., $\bar{p} / \pi$ ratio) etc. One may hope that they will be resolved in the near future within the same set of adjusted parameters. That would provide deeper understanding of collective thermodynamical and mechanical properties of the bulk matter and its evolution. 


\section{$5 \quad$ Parton energy loss in micro-QCD}

Experimentalists observe collimated groups of particles called jets created in high energy collisions. There are different stages of jet formation:

- Production of the initial high energy parton (described by pQCD).

- The partonic stage of jet evolution in the medium with account of external fields (described by DGLAP equations with account of the LPM-effect).

- Hadronization - final particles production (using the parton distribution functions known from experiment).

Two parameters are especially crucial for such description: $\hat{q}=\left\langle p_{\perp}^{2}\right\rangle / \lambda$ and $L$-the medium size. as well as their combination $\left(\omega_{c}=\frac{1}{2} \hat{q} L^{2}\right)$.

The physical problem at hand is the computation of the leading contribution to the medium-induced energy loss $\Delta E$ of the parton produced inside the medium on its way from the hot dense fireball. Generically the energy loss $\Delta E$ depends on the medium thickness $L$ and the bulk characteristics of the medium and is described by some probability distribution $\mathcal{P}(\Delta E)$.

In the general case the losses are determined by the following factors:

- Probability of an elementary event leading to energy loss is characterized by the opacity $N=L / \lambda$, where $\lambda$ is the mean free path of the parton in the medium under consideration. For the particle with integrated particle-medium interaction cross section $\sigma$ and the medium density $\rho$ the mean free path can be estimated as $\lambda \sim 1 /(\rho \sigma)$.

- The intensity of the impact or scattering power of the medium is characterized by the transport coefficient $\hat{q}=\left\langle p_{\perp}^{2}\right\rangle / \lambda$, where $\left\langle p_{\perp}^{2}\right\rangle$ is the average transverse momentum squared that the propagating particle gets from the elementary act of collision. In the thermal medium $\hat{q}=m_{D}^{2} / \lambda$ where $m_{D}$ is the Debye mass.

At present there exists a general consensus, supported by extensive model calculations, that the radiative medium-induced energy loss is the dominant one, see e.g. [8]. Theoretical work on studying the properties of mediuminduced gluon radiation was carried out by several groups [9, 10, 11, 12, 13, 14, 15, 16, 17]. The approaches of the above-listed groups differ in details of 
treating the relevant kinematics of the radiation and the details of describing the medium under consideration.

Unfortunately, variations of $p_{\perp}$ per unit length, described by the parameter $\hat{q}$, differ sometimes by an order of magnitude in different models when compared with experiment.

In the limit $\omega \ll \omega_{c}$ the medium acts coherently reducing the intensity of radiation while that of $\omega \gg \omega_{c}$ corresponds to incoherent scattering.

$$
\begin{aligned}
&\left.\omega \frac{d I}{d \omega}\right|_{\omega \ll \omega_{c}} \approx \alpha_{s} \sqrt{\frac{\hat{q} L^{2}}{\omega}}, \\
&\left.\omega \frac{d I}{d \omega}\right|_{\omega \gg \omega_{c}} \approx \alpha_{s} \frac{\hat{q} L^{2}}{\omega} .
\end{aligned}
$$

In the soft limit $\omega \ll \omega_{c}$ one has for the average energy loss

$$
\langle\Delta E\rangle \bumpeq \int_{0}^{\omega_{c}} d \omega \omega \frac{d I}{d \omega} \sim \hat{q} L^{2}
$$

For some time one thought that the quadratic dependence on the medium thickness $L$ is a specifically non-Abelian effect. Recently, however, in [18, see also [19], it was shown that the effect is generic and is valid both in QED and QCD for the radiation of particle produced inside the medium at the initial piece of its trajectory.

Thus the finite length is crucial. The key question is therefore to choose, for describing the in-medium QCD cascade, the language allowing a credible space-time interpretation. As emphasized in [20], the only scheme allowing such interpretation is the old-style PYTHIA virtuality-ordered cascade where connection to the spatiotemporal pattern is achieved by calculating the lifetime $\tau$ of a virtual parton which, for a parton with the energy $E$ and virtuality $Q^{2}$ that has been created in the decay of its parent parton with the virtuality $Q_{\text {par }}^{2}$, reads

$$
\tau=E\left(\frac{1}{Q^{2}}-\frac{1}{Q_{\mathrm{par}}^{2}}\right) .
$$

Another important ingredient is the statement about the angular ordering. While valid in vacuum, it can be modified for in-medium cascades. It has been shown in [21] that the properties of the cascade can be drastically changed if these problems are properly treated. 


\section{The macroscopic approach to the quark- gluon medium}

Macroscopic collective properties of a medium may reveal themselves in its mechanical motion as a whole (e.g., viscosity) described by hydrodynamics or in its response to external color currents (e.g., chromopermittivity) described by in-medium QCD.

The medium itself can radiate in response to permutations. The genuine role of the medium and its collective properties are most clearly revealed by its polarization $\mathbf{P}$ due to the external current. The macroscopic approach to the description of such collective properties is the most suitable one. The linear response of the medium to the electromagnetic field $\mathbf{E}$ is usually described as

$$
\mathbf{P}=\frac{\epsilon-1}{4 \pi} \mathbf{E}
$$

where $\epsilon$ is the dielectric (in macro-QED) or chromoelectric (in macro-QCD) permittivity. It is seen that the polarization can be quite strong for large values of $\epsilon$. The initial radiation process serves as a trigger for the collective response of the medium initiated by the polarization. The well known examples are provided by the Cherenkov radiation, the wake and the transition radiation.

What is typical for their description is the (approximate) constancy of the particle velocity vector used in the external current. In ultrarelativistic processes $(\gamma \gg 1)$ the relative change of the velocity is much smaller than the relative energy loss because they are connected by the formula

$$
\frac{\Delta E}{E}=\left(\gamma^{2}-1\right) \frac{\Delta v}{v}
$$

and the above statement is well supported. The velocity loss can become noticeable only for non-relativistic partons.

In what follows we consider very high energy processes. It is well known that the gluons become the main component of the wave functions of the colliding hadrons. At LHC, the $g g$-luminosity is at least by an order of magnitude higher than the $\sum q q$-luminosity. Therefore the in-medium gluodynamics [22] is considered below. It simplifies the formulae. Quarks can be easily included too [23].

The in-medium equations of gluodynamics differ from the in-vacuum equations by introducing a chromopermittivity of the quark-gluon medium. 
Similar to the dielectric permittivity in electrodynamics it describes the linear response of the matter to passing partons.

Analogously to electrodynamics, one can treat the linear response of the medium by the medium permittivity $\epsilon$ if $\mathbf{E}$ is replaced by $\mathbf{D}=\epsilon \mathbf{E}$ in $F^{\mu \nu}$.

Then, in terms of potentials the equations of in-medium gluodynamics are cast in the form [22] (for $\epsilon=$ const)

$$
\begin{gathered}
\triangle \mathbf{A}_{a}-\epsilon \frac{\partial^{2} \mathbf{A}_{a}}{\partial t^{2}}=-\mathbf{j}_{a}-g f_{a b c}\left(\frac{1}{2} \operatorname{curl}\left[\mathbf{A}_{b}, \mathbf{A}_{c}\right]+\epsilon \frac{\partial}{\partial t}\left(\mathbf{A}_{b} \Phi_{c}\right)+\frac{1}{2}\left[\mathbf{A}_{b} \text { curl } \mathbf{A}_{c}\right]-\right. \\
\left.\epsilon \Phi_{b} \frac{\partial \mathbf{A}_{c}}{\partial t}-\epsilon \Phi_{b} \operatorname{grad} \Phi_{c}-\frac{1}{2} g f_{c m n}\left[\mathbf{A}_{b}\left[\mathbf{A}_{m} \mathbf{A}_{n}\right]\right]+g \epsilon f_{c m n} \Phi_{b} \mathbf{A}_{m} \Phi_{n}\right),(10) \\
\triangle \Phi_{a}-\epsilon \frac{\partial^{2} \Phi_{a}}{\partial t^{2}}=-\frac{\rho_{a}}{\epsilon}+g f_{a b c}\left(-2 \mathbf{A}_{c} \operatorname{grad} \Phi_{b}+\mathbf{A}_{b} \frac{\partial \mathbf{A}_{c}}{\partial t}-\epsilon \frac{\partial \Phi_{b}}{\partial t} \Phi_{c}\right)+ \\
g^{2} f_{a m n} f_{n l b} \mathbf{A}_{m} \mathbf{A}_{l} \Phi_{b} .
\end{gathered}
$$

If one neglects the terms with explicitly shown charge $g$ in Eqs. (10), (11), one gets the set of abelian equations, which differ from electrodynamical equations by the color index $a$ only. The most important property of the solutions of these equations is that while the in-vacuum $(\epsilon=1)$ equations do not admit any radiation processes, for $\epsilon \neq 1$ there appear solutions of these equations with non-zero Poynting vector even in the classical approach. They predict such coherent effects as Cherenkov gluons, the wake and transition radiation. This corresponds well to the microscopic description in which the matter, at early times after collision, is described in terms of the coherent classical field.

There are several classical polarization effects in the quark-gluon medium wherefrom one can determine its chromodynamical properties. Actually, this idea was first promoted in [24, 25] relying on the similarity of quarks to electrons and gluons to photons. The emission of Cherenkov gluons analogous to Cherenkov photons was predicted and used for interpretation of a cosmic ray event where a ring-like structure was just observed [26] reminding Cherenkov findings of photon rings. The strong support this idea got, however, much later from RHIC data on nucleus-nucleus collisions.

Other effects include the possible role of Cherenkov gluons in asymmetry of shapes of resonances traversing the quark-gluon medium and the wake radiation producing the shift in azimuthal distributions in non-central collisions. I describe briefly only the ring effect referring to [1] for more details. 
This effect is derived from the classical solutions of Eqs (10), (11) if the current with constant velocity $\mathbf{v}$ along the $z$-axis is considered:

$$
\mathbf{j}(\mathbf{r}, t)=\mathbf{v} \rho(\mathbf{r}, t)=4 \pi g \mathbf{v} \delta(\mathbf{r}-\mathbf{v} t) .
$$

These solutions describe the cone-like emission of Cherenkov gluons at the typical angle

$$
\cos \theta=\frac{1}{v \sqrt{\epsilon}}
$$

It is constant for constant $\epsilon>1$.

Let us stress that this classical effect has collective non-perturbative origin even though $\alpha_{S}$ enters seemingly linearly. The chromopermittivity $\epsilon$ hides the non-perturbative terms responsible for the collective medium response.

Similar to electrodynamics [27], the energy-angular spectrum of emitted gluons [28, 29] per the unit length

$$
\frac{d N^{(1)}}{d \Omega d \omega}=\frac{\alpha_{S} C \sqrt{x}}{2 \pi^{2}}\left[\frac{(1-x) \Gamma_{t}}{\left(x-x_{0}\right)^{2}+\left(\Gamma_{t}\right)^{2} / 4}+\frac{\Gamma_{l}}{x}\right],
$$

where

$$
x=\cos ^{2} \theta, x_{0}=\epsilon_{1 t} /\left|\epsilon_{t}\right|^{2} v^{2}, \quad \Gamma_{j}=2 \epsilon_{2 j} /\left|\epsilon_{j}\right|^{2} v^{2}, \quad \epsilon_{j}=\epsilon_{1 j}+i \epsilon_{2 j} .
$$

The real $\left(\epsilon_{1}\right)$ and imaginary $\left(\epsilon_{2}\right)$ parts of $\epsilon$ are taken into account.

It is clearly seen from Eq. (14) that the transverse and longitudinal parts of the chromopermittivity are responsible for the distinctly different effects. The ringlike Cherenkov structure (conical emission) is exhibited in the first term of (14). The second term defined by the longitudinal part of $\epsilon$ is in charge of the wake radiation. It is important that both of them are independent on parton masses. This fact is supported by experiment where there is no difference between energy losses of light and heavy quarks.

The first term was fitted [28] to get the values of real $\left(\epsilon_{1} \approx 6\right)$ and imaginary $\left(\epsilon_{2} \approx 0.8\right)$ parts of the chromopermittivity. The fit to experimental data (with elliptic flow subtracted) is shown in Fig. 1.

These plots show the projection of rings on their diameter just as was done in original papers by Cherenkov in 1937. It is the very first determination of the chromopermittivity from experiment in the definite range of the transverse momentum of created particles. In principle, the chromopermittivity may depend on it (the well known dispersion in electrodynamics). For discussion of this problem and other effects see [1]. Unfortunately, I had to omit many figures from original presentation and refer the readers to it. 

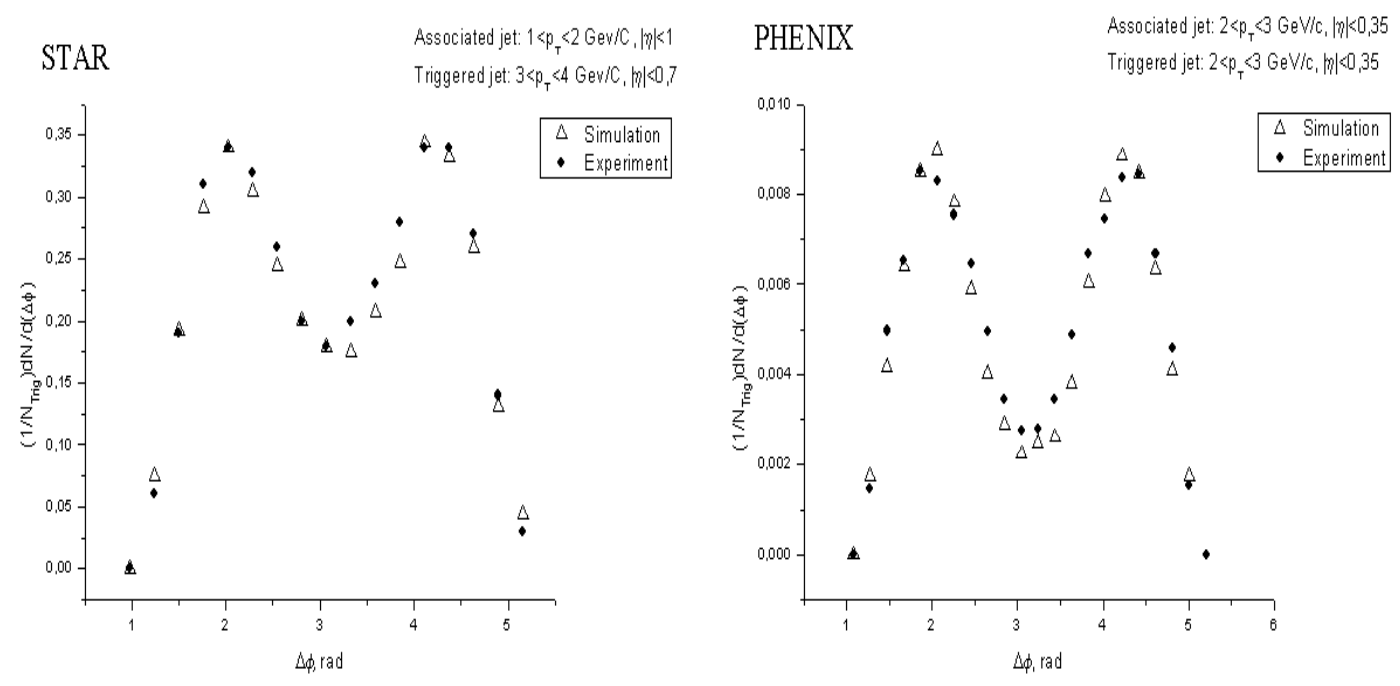

Figure 1: Associated azimuthal correlations at STAR and PHENIX: circles - experiment, triangles- theory.

\section{Conclusions}

Let us briefly summarize what have we learned about the matter properties from comparison of experimental data with hydrodynamics and QCD.

\section{HYDRODYNAMICS:}

temperature and energy density evolution, azimuthal (elliptic) flow, viscosity, transfer properties, QGP lifetime, baryon density, hadrocomposition.

\section{QCD:}

micro-approach - jet quenching, the transfer coefficient $\hat{q}$ (LPM-effect), QCD-vertex, role of angular ordering, color and medium size.

macro-approach - chromopermittivity, parton density, energy loss to Cherenkov gluons, free path length of partons, shape of resonances passing through the medium, the wake, independence on quark masses.

\section{Acknowledgements}

This work was supported by RFBR grants 09-02-00741; 08-02-91000CERN and by the RAN-CERN program. 


\section{References}

[1] Dremin I M , Leonidov A V Physics-Uspekhi 53 N11 (2010)

[2] Jalilian-Marian J, Kovner A, Leonidov A, Weigert H Phys. Rev. D 59 034007 (1999); Iancu E, Leonidov A, McLerran L D Nucl. Phys. A 692 $583(2001)$

[3] Shuryak E V Prog. Part. Nucl. Phys. 6248 (2009)

[4] Heinz U arXiv:hep-ph/0407360; CERN-2004-001

[5] Yukalov V I, Yukalova E P Physica A 243382 (1997)

[6] Shuryak E V, Zahed I Phys. Rev. D 70054507 (2004)

[7] Rapp R et al Nucl. Phys. A 830 861c (2009)

[8] Zakharov B G JETP Lett. 86444 (2007)

[9] Baier R et al. Phys. Lett. B 345277 (1995);

[10] Djordjevic M, Gyulassy M, Wocks S, Phys. Rev. Lett. 94112301 (2005)

[11] Wiedemann U A Nucl. Phys. B 588303 (2000)

[12] Armesto N, Salgado C A, Wiedemann U A Phys. Rev. D 69114003 (2004)

[13] Zakharov B G JETP Lett 63952 (1996)

[14] Zakharov B G JETP Lett 65615 (1997);

[15] Zakharov B G JETP Lett. 70176 (1999)

[16] Guo X F, Wang X N Phys. Rev. Lett. 853591 (2000);

[17] Arnold P, Moore G D, Yaffe L G JHEP 11057 (2001);

[18] Peigne S, Smilga A Phys. Usp. 52659 (2009)

[19] Zakharov B G JETP Lett. 801 (2004) 
[20] Zapp K, Ingelman G, Rathsman J, Stachel J, Wiedemann U A Eur. Phys. J C 60617 (2009)

[21] Leonidov A V, Nechitailo V A, arXiv:1006.0366 [nucl-th]

[22] Dremin I M Eur. Phys. J. C 5681 (2008)

[23] Djongolov M K, Pisov S, Rizov V J. Phys. G 30425 (2004)

[24] Dremin I M JETP Lett. 30140 (1979)

[25] Dremin I M Sov. J. Nucl. Phys. 33726 (1981)

[26] Apanasenko A V et al. JETP Lett. 30145 (1979)

[27] Grichine V M Nucl. Instr. Meth. A 482629 (2002)

[28] Dremin I M, Kirakosyan M R, Leonidov A V, Vinogradov A V Nucl. Phys. A 826190 (2009)

[29] Dremin I M Mod. Phys. Lett. A 25591 (2010) 